\title{
Response to "Prognostic Value of Epicardial Fat Thickness as a Biomarker of Increased Inflammatory Status in Patients with Type 2 Diabetes Mellitus and Acute Myocardial Infarction"
}

\author{
Evangelos K. Oikonomou, Charalambos Antoniades \\ Division of Cardiovascular Medicine, University of Oxford, Oxford, United Kingdom
}

To the editor

We read with great interest the article entitled "Prognostic Value of Epicardial Fat Thickness as a Biomarker of Increased Inflammatory Status in Patients with Type 2 Diabetes Mellitus and Acute Myocardial Infarction" by Opincariu et al..$^{1}$ In their study, the authors demonstrate that higher epicardial fat thickness (EFT) correlates with persistence of systemic inflammation, more severe ventricular remodelling and impairment of ventricular function following acute myocardial infarction (AMI) in patients with type 2 diabetes mellitus. This is an interesting study which highlights the role of the epicardial fat depot as a key player in the pathophysiology of inflammation and cardiac disease.

However, the current study fails to acknowledge several limitations associated with the measurement of EFT on 2-dimensional echocardiography. First, even though there is currently no clear consensus, most authors agree that EFT should be measured on the right ventricular free wall in at least two locations, from both parasternal longitudinal and transverse parasternal views and at three consecutive beats using specific anatomical landmarks, such as the aortic annulus. ${ }^{2,3}$ Based on the "methods" section, it is not clear whether the authors in this particular study used a specific protocol to ensure the validity and reliability of their measurements. Second, EFT and epicardial fat volume (EFV) measurements using echocardiography or computed tomography (CT) fail to take into account differences in body size or total obesity. It is therefore imperative that such biomarkers are adjusted for differences in body size (e.g. indexed for body surface area) and that obesity markers such as body mass index are included in multivariate regression models. Even in EFV measurements on CT, non-correction for body size can lead to misleading results and as a result, EFV values are usually adjusted for body surface area. ${ }^{4}$ Third, the terms epicardial fat "thickness" and "volume" should not be used interchangeably. In fact, EFT as measured at the long parasternal axis view does not necessarily correlate with total EFV, since linear measurements at one site may not reflect accurately the distribution and overall volume of the epicardial fat. In other words, EFT measured on echocardiography is not considered a surrogate biomarker for total EFV. ${ }^{5}$ Finally, one study has demonstrated poor reproducibility for EFT measurements using echocardiography, as well as poor correlation with measurements derived from high-resolution multide- 
tector CT. ${ }^{3}$ Overall, these findings question the validity of echocardiography as a reliable method for assessment of EFT and volume.

In summary, these remarks highlight some significant and frequently encountered issues in studies that assess EFT. Given the absence of consensus on the correct way to measure EFT and volume on echocardiography, there is large heterogeneity in the methods described in the literature. The lack of a detailed and reproducible protocol for assessment of these indices, as well as the lack of adjustment for possible confounders such as body size and obesity limit the external validity of such studies and emphasise the need for a more standardised approach to non-invasive imaging of adipose tissue depots.

\section{CONFLICT OF INTEREST}

None declared.

\section{REFERENCES}

1. Opincariu D, Mester A, Dobra M, et al. Prognostic Value of Epicardial Fat Thickness as a Biomarker of Increased Inflammatory Status in Patients with Type 2 Diabetes Mellitus and Acute Myocardial Infarction. Journal of Cardiovascular Emergencies. 2015;1(2):11-18. DOI: 10.1515/jce-2016-0003.

2. Iacobellis G, Willens HJ. Echocardiographic epicardial fat: a review of research and clinical applications. J Am Soc Echocardiogr. 2009;22(12):1311-1319. quiz 417-8. DOI: 10.1016/j.echo.2009.10.013.

3. Saura D, Oliva MJ, Rodriguez D, et al. Reproducibility of echocardiographic measurements of epicardial fat thickness. Int J Cardiol. 2010;141(3):311-313. DOI: 10.1016/j. ijcard.2008.11.127.

4. Lu MT, Park J, Ghemigian K, et al. Epicardial and paracardial adipose tissue volume and attenuation - Association with highrisk coronary plaque on computed tomographic angiography in the ROMICAT II trial. Atherosclerosis. 2016;251:47-54. Epub 2016/06/09. doi: 10.1016/j.atherosclerosis.2016.05.033. PubMed PMID: 27266821.

5. Talman AH, Psaltis PJ, Cameron JD, Meredith IT, Seneviratne SK, Wong DT. Epicardial adipose tissue: far more than a fat depot. Cardiovasc Diagn Ther. 2014;4(6):416-429. DOI: 10.3978/j.issn.2223-3652.2014.11.05. 